\title{
Development and Performance Evaluation of a Computer Program Based on Neural Network Mathematical Models for Forecasting By- Product Yield
}

\author{
Yelena Vasileva $^{1}$, Aleksandr Nevedrov $^{1}$, and Sergey Subbotin ${ }^{1,2}$ \\ ${ }^{1}$ T.F. Gorbachev Kuzbass State Technical University, 650000, Russian Federation \\ ${ }^{2}$ Koks PJSC, 6 1st Stakhanovkaya, Kemerovo, 650021, Russian Federation
}

\begin{abstract}
Process performance of coking plants are based on data on the yield of by-products of coking coal and their quality, therefore, much attention is paid to the issues of their analysis. In view of the complexity and insufficient knowledge of the relationship between these parameters, mathematical modeling of this dependence using neural networks is of great interest. Based on a mathematical analysis of experimental data on the quality indicators of coal, coal concentrates and the by-product yield, neural network mathematical models have been developed to forecast the parameters under study. The neural network is based on the Ward's network. Based on the results of the research, the application "Intelligent Information System for Forecasting By-product Yield" was created, which implements neural networks [1]. The relative forecasting error for the parameter "coke" is $0.64 \pm 0.23 \%$, "coal tar" is $19.53 \pm 5.25 \%$, "crude benzene" is $10.02 \pm 2.83 \%$, and "coke gas" is $5.11 \pm 1.34 \%$. A comparative analysis of the data obtained using the developed design method is carried out, with the simulation results using existing methods, as well as with the production values of by-products yield.
\end{abstract}

\section{Introduction}

By-product yield is a fundamental factor in planning and evaluation of the operation of byproduct coking enterprises, therefore, the issue of forecasting this parameter is fundamental in planning cost-performance ratio of production. Since the 1920s, a large number of dependences of the yield of various by-products on the coal quality coefficient, from the volatile-matter yield to methods based on a modern understanding of their structure and properties, determined on the basis of data obtained during petrographic and ultimate analyzes [2-4], including with the calculation of the chemical potential of coal $[5,6]$.

To determine the by-product yield, a large number of dependencies have been proposed, in which indicators of the proximate analysis of coal or coal charge are used as the basis for forecasting. A large number of formulas are based on indicators of the volatile-matter yield on various states of fuel. This is explained by the fact that these parameters, along with indicators characterizing coking index, determine the possibility of using the studied coal in the technological process [7], as well as the yield of the basic product of this process - bulk 
coke and the entire gamut of by-products $[8,9]$. In addition to the considered indicators, various scientists can additionally introduce parameters into the equations that take into account the yield of volatile matter from coke or characterize the thermal behavior of the process [10].

Due to the complexity of determining the dependence of the by-product yield on the quality coefficient of coal and coal charge, the application of the neural network method of mathematical modeling to solve the problem of their forecasting is of the most interest. The development of a method for forecasting the by-product yield based on neural network mathematical models was carried out on the basis of the Department of Chemical Technology of Solid Fuels of the Institute of Chemical and Oil and Gas Technologies and the Department of Applied Information Technologies of the Institute of Information Technologies, Engineering and Motor Transport of T.F. Gorbachev Kuzbass State Technical University together with Koks PJSC in Kemerovo.

The aim of the work is to develop a method for forecasting the yield of by-products from coal and coal blends, using models developed with the application neural networks.

\section{Materials and methods}

The studies were based on the analysis of samples of coal and coal concentrates of Koks PJSC, taken from the batches of fuel supplied to the enterprise. The choice of the determined quality indicators was based on the possibility of using the developed mathematical models to predict the by-product yield in the operation of enterprises whose activities are associated with coal mining and processing. Proceeding from this, it is most correct to apply methods for assessing the quality indicators of coal used in the laboratories of enterprises producing and coking coal. The main methods used in the study of the quality of coal are on dry ash test of fuel in accordance with ISO 1171: 2010, the volatile-matter yield on various states of fuel in accordance with ISO 562: 2010, ISO 5071-1: 2013, total sulfur in accordance with ISO 351-96, plastometric indicators according to GOST 1186-87, the expansion index according to the IGI-DMetI method according to GOST 14056-77, the free swell index according to ISO 501-81, petrographic indicators (an arbitrary vitrinite reflectance index in immersion, the content of vitrinite and the sum of fusenized (leaning) components) by ISO 7404-3:2009, ISO 7404-5-85, total carbon and hydrogen contents according to ISO 625-75. Based on the obtained values of the elemental composition of the organic mass of coal, characteristics are calculated that characterize the features of the molecular structure of coal and affect the by-product yield and composition.

\section{Results and discussion}

Neural networks are a universal tool for solving the forecasting problem, however, at present, there are no universal tools developed using them. Based on the obtained quality indicators of the studied fuel samples and the by-products yield [11], as well as the analysis of the data of the studies using the statistical methods given in [12], which showed the nonlinearity of the relationship between the input and forecast parameters, a decision was made to develop and implement a Ward's network designing its own neural network architecture. The topology of the standard Ward's network used in studies consists in splitting neurons into groups with different transfer functions, which allows finding more complex nonlinear dependencies [13].

Designing a neural network for forecasting the parameter "Coke" is presented in Fig. 1, for the out parameter "Coal tar" - in Fig. 2, for the out parameter "Crude benzene" - in Fig. 3 , for the out parameter "Coke gas" - in Fig. 4. 


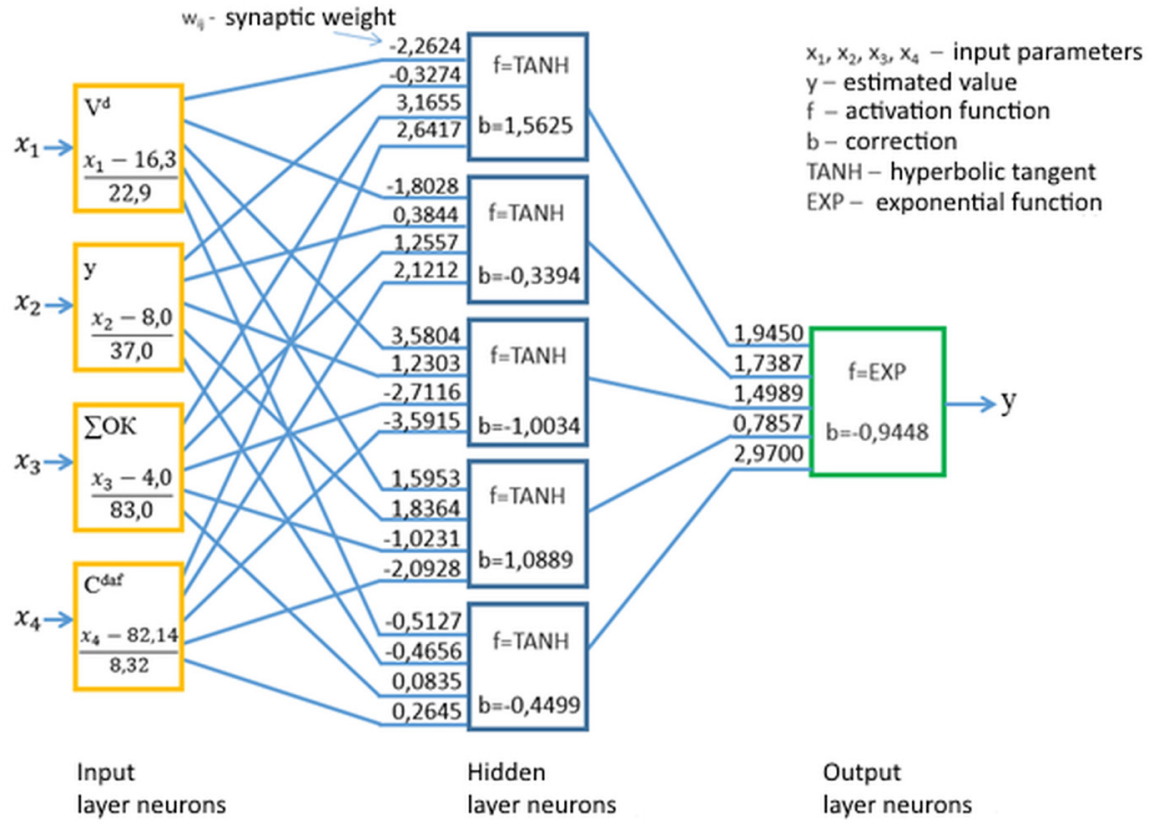

Fig. 1. Designing a neural network for the out parameter "Coke".

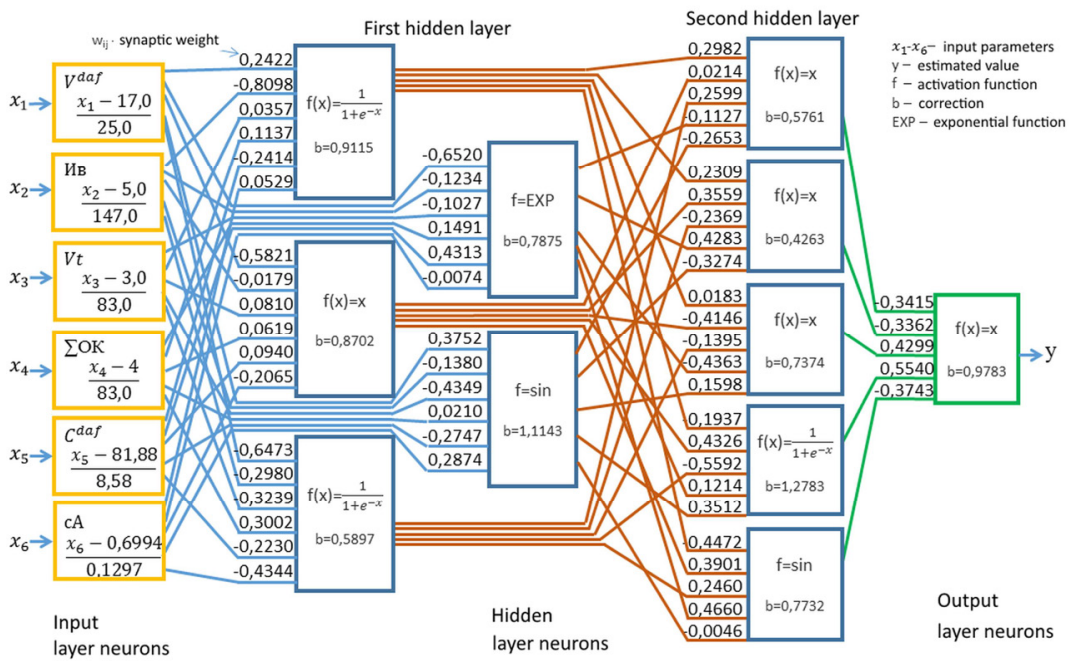

Fig. 2. Designing a neural network for the out parameter "Coal tar". 


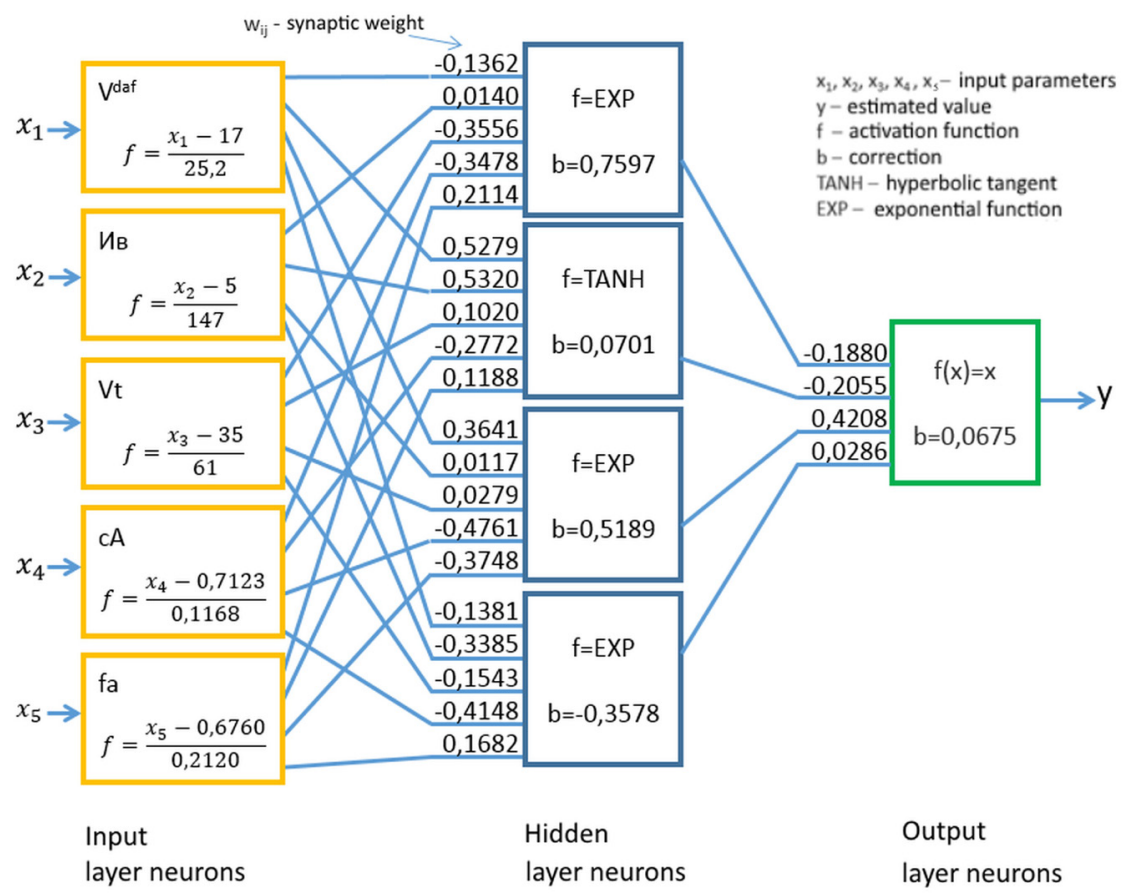

Fig. 3. Designing a neural network for the out parameter "Crude benzene".

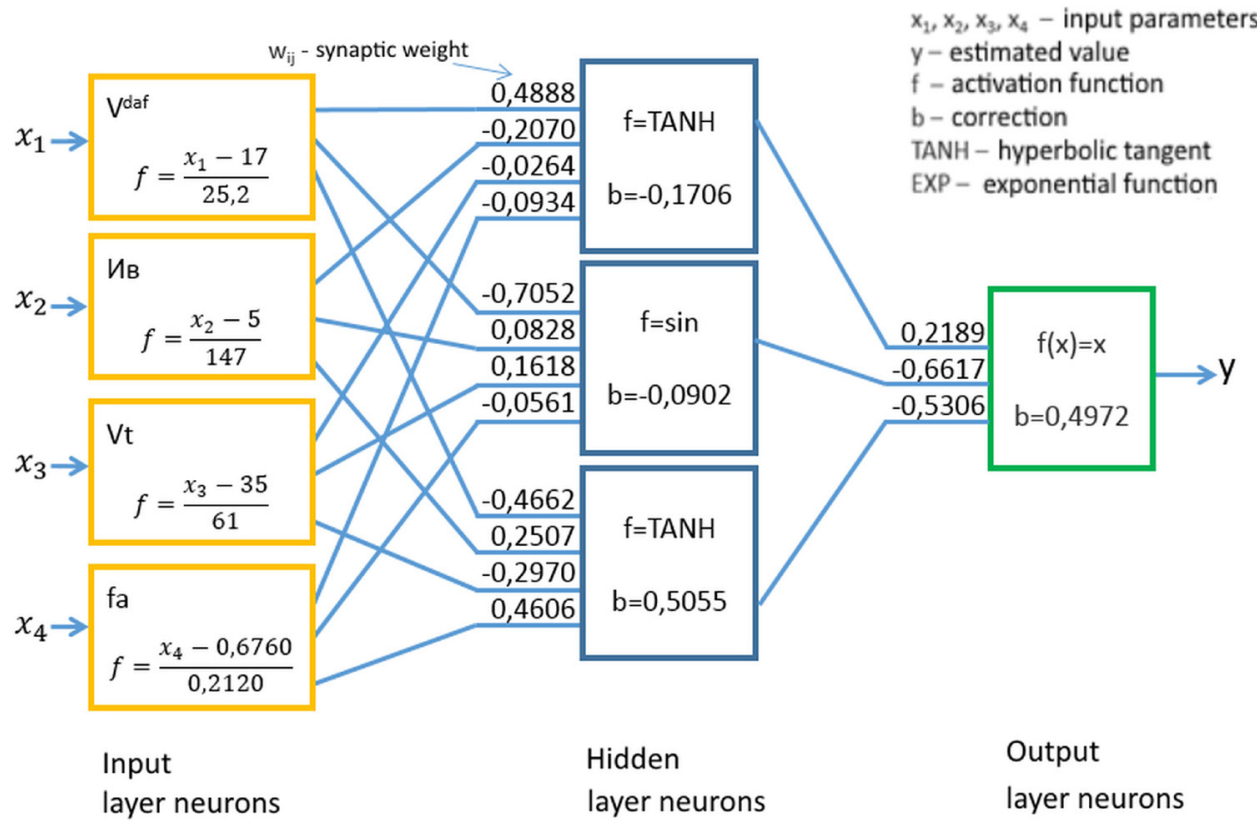

Fig. 4. Designing a neural network for the out parameter "Coke gas". 
Developed on the basis of research results, the computer program "Intelligent Information System for Forecasting By-product Yield" [1] is divided into two functional parts. In the first part, the operator creates and trains neural networks; in the second one, the operator forecasts using the neural networks created in the first part.

The program window for designing a network can be divided into the following parts: at the top there are buttons and fields for the input parameters necessary for creating, training and saving the network, and at the bottom there are graphs reflecting the behavior of the network during training (Fig. 5).

When training a neural network, the window on the left graphically displays the change in the value of the average relative error. The abscissa axis in this case characterizes the numbers of epochs, and the values of the average relative error are shown on the ordinate axis. The theoretical response (yellow markers) and the response obtained using the neural network (blue markers) are graphically displayed on the right.

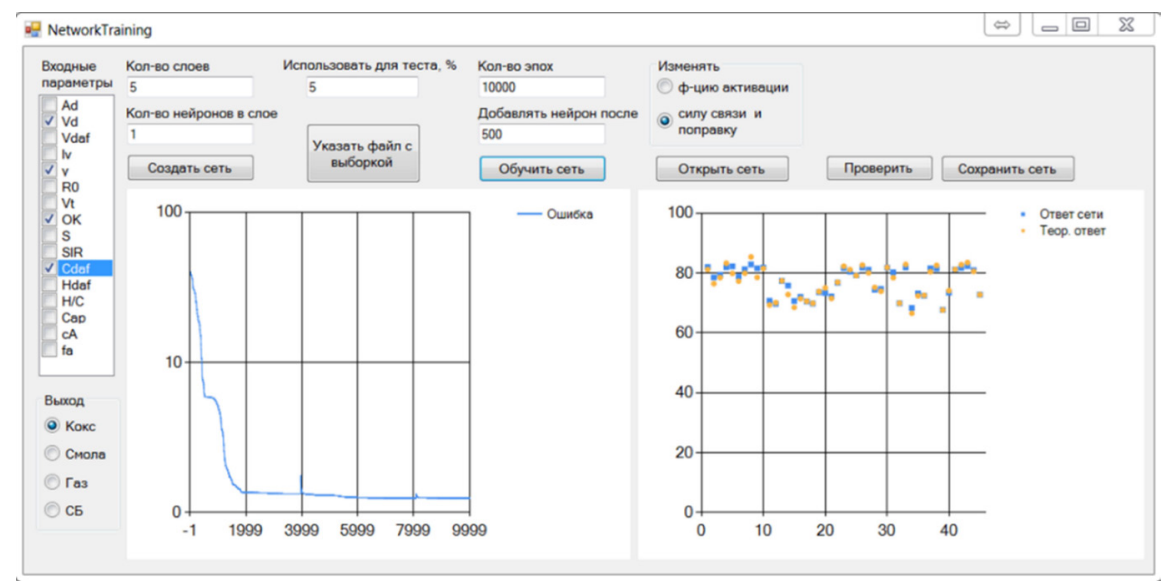

Fig. 5. Network training program window.

At the top of the "Main form of the user application" window (Fig. 6), you can see the average relative error of the networks. There are the input fields for input parameters under this window. Next, there is a block for displaying forecasted values. The "Clear" button deletes previously entered values from the fields of the "Input Parameters" form. The "Calculate" button starts the operation of neural networks, the result of which is displayed in the "Response" block. The values obtained are the forecasted values of the by-products yield. 


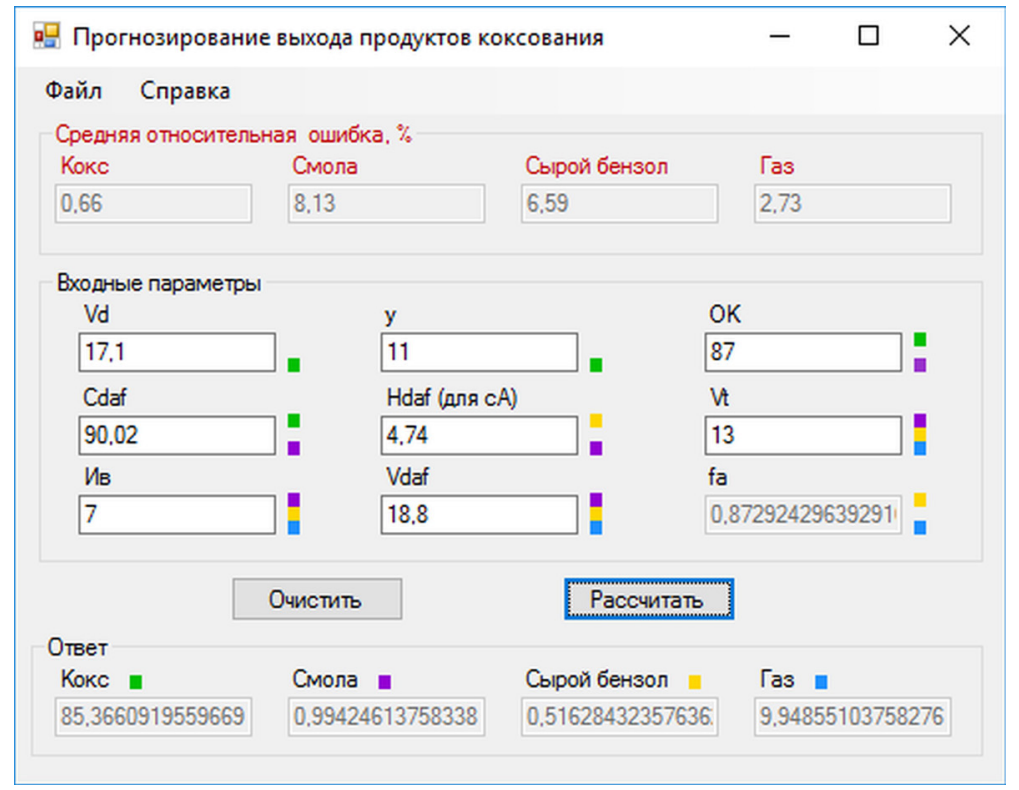

Fig. 6. The window of the main form of the user application of the program.

\section{Conclusion}

The fundamental possibility of using the method of artificial neural networks in determining the relationship between the basic by-products yield and the coal quality, characterized by standard parameters, as well as the development of a forecast method based on the found dependencies, are shown in the paper. When applying the research methods in the factory laboratories of by-product coking enterprises, when developing the model, it becomes possible to work with it for a preliminary assessment of charge coal and the calculation of the by-product yield in the preparation of coal charge. The relative error of the values calculated using the developed forecast method, compared with the production indicators of the by-product yield by the example of coke, coal tar and crude benzene, is approximately the same throughout the entire observation period and is in the range of the error determined for the method: for the parameter "coke" - $0.64 \pm 0.23 \%$, "coal tar" $19.53 \pm 5.25 \%$, "crude benzene" - 10.02 $\pm 2.83 \%$, "coke gas" - $5.11 \pm 1.34 \%$

The by-product yield values were forecasted according to this model without taking into account the influence of the coking process conditions, which determines the direction of further research in this area, based on the study of their influence on the by-product yield, which may reduce the relative forecast error. The difficulty in obtaining an adequate model is the presence of many influencing factors and their inconstancy within the limits of both the coke oven battery and by-product coking.

\section{References}

1. E. V. Vasileva, A. B. Pileckaya, V. S. Doroganov, T. G. Cherkasova, S. P. Subbotin, A. V. Nevedrov, E. A. Koshelev, N. G. Kolmakov, Certificate 2017662199 Russian Federation. Certificate of registration of a computer program. Intelligent information system for predicting the yield of coking products (Rospatent, Moscow, 2017) 
2. V. N. Rubchevskij, Yu. A. Chernyshov, S. A. Ovchinnikova, A. I. Kompaniets, I. D. Drozdnik, D. V. Miroshnichenko, M. B. Golovko, E. B. Pribavkina, Coke and Chemistry, 52, 4 (2009)

3. Yu. V. Stepanov, G. M. Karpin, Coke and Chemistry, 52, 10 (2009)

4. A. B. Danilov, G. S. Verdibozhenko, I. D. Drozdnik, D. V. Miroshnichenko, Yu. S. Kaftan, M. B. Golovko, Coke and Chemistry, 55, 11 (2012)

5. S. G. Gagarin, Coke and Chemistry, 43, 3 (2000)

6. M. B. Golovko, I. D. Drozdnik, D. V. Miroshnichenko, Yu. S. Kaftan, Coke and Chemistry, 55, 6 (2012)

7. S. G. Gagarin, Coke and Chemistry, 43, 11-12 (2000)

8. I. D. Drozdnik, Yu. S. Kaftan, S. D. Menshikova, Coke and Chemistry, 33, 8 (1990)

9. Yu. V. Teleshev, S. I. Kaufman, M. S. Sheptovickij, Coke and Chemistry, 40, 1 (1997)

10. P. N. Gorelov, M. S. Kotelenec, Coke and Chemistry, 30, 1 (1987)

11. E. V. Vasileva, T. G. Cherkasova, N. G. Kolmakov, S. P. Subbotin, E. A. Koshelev, A. V. Nevedrov, A. V. Papin, Coke and Chemistry, 58, 11 (2015)

12. E. V. Vasileva, V. S. Doroganov, A. B. Piletskaya, T. G. Cherkasova, A. G. Pimonov, N. G. Kolmakov, S. P. Subbotin, A. V. Nevedrov, A. V. Papin, Coke and Chemistry, 60, 9 (2017)

13. T. M. Martinetz, S. G .Berkovich, K. J. Schulten, IEEE Transactions on Neural Networks, 4, 4 (1993) 